\title{
Structure of metal nanowires in nanoporous alumina membranes studied by EXAFS and X-ray diffraction
}

\author{
R.E. Benfield ${ }^{1, a}$, D. Grandjean ${ }^{1}$, J.C. Dore ${ }^{1}$, Z. Wu ${ }^{1, b}$, M. Kröll ${ }^{2, c}$, T. Sawitowski², and G. Schmid ${ }^{2}$ \\ 1 Centre for Materials Research, School of Physical Sciences, University of Kent, Canterbury CT2 7NR, UK \\ 2 Institut für Anorganische Chemie, Universität GH Essen, Universitätsstr. 5-7, D-45117 Essen, Germany
}

Received 30 November 2000

\begin{abstract}
The structure of nanowires of different metals grown within nanoporous alumina membranes has been studied by EXAFS, WAXS and high energy X-ray diffraction. Nanowires of gold, silver, copper and iron adopt the lattice structure and bond distances of the bulk metals. Cobalt nanowires on the other hand were composed of a mixture of hcp phase, stable at room temperature, and fcc phase, which in bulk cobalt is normally stable only at high temperatures, in a ratio depending on the pore size. The nanowires are non-continuous but are made of nanocrystallites whose shape and size was found to depend strongly on the metal. All the metals except gold showed the presence of a preferred orientation which was slight in the case of $\mathrm{Ag}$ and $\mathrm{Cu}$ but much stronger in the case of iron and cobalt nanowires.
\end{abstract}

PACS. 61.10.Ht X-ray absorption spectroscopy: EXAFS, NEXAFS, XANES, etc. - 61.10.-i X-ray diffraction and scattering $-61.46 .+\mathrm{w}$ Nanoscale materials: clusters, nanoparticles, nanotubes, and nanocrystals

\section{Introduction}

There is a remarkable trend in materials research to prepare and characterise nano-materials of quantum-dot size in regular arrangements [1]. For instance, self-assembled nanoparticles of magnetic transition metals have recently attracted attention because of their potential use as new information storage devices [2]. A promising way to achieve a regular array of nano-materials is to use nanoporous alumina membranes [3] as a template. These membranes have a regular array of pores which can be controlled by varying the conditions of preparation. They are ideally suited as a matrix to position materials such as carbon nanotubes [4], metal clusters and colloids [5] or metal nanowires in confinements with regular spacings.

In the framework of our EU-funded research network CLUPOS [6] we have prepared a series of $\mathrm{Au}, \mathrm{Ag}, \mathrm{Cu}$, $\mathrm{Fe}$ and Co nanowires within nanoporous alumina membranes of pore size varying from $c a .5$ to $72 \mathrm{~nm}$. These nano-materials represent isolated one-dimensional quantum wires with prospective applications in electronic devices [7]. The physical properties of these nano-materials are different from the bulk form. For instance the Fe and Co samples feature unusual ferromagnetic properties [8].

\footnotetext{
${ }^{\mathrm{a}}$ e-mail: R.E.Benfield@ukc.ac.uk

b Permanent address: BSRL, Institute of High Energy Physics, Chinese Academy of Sciences, P.O. Box 918, Bin 2-7, Beijing 100039, P.R. China.

c Present address: Physics Department, Trinity College Dublin, Dublin 2, Ireland.
}

To achieve a better understanding of the physical properties of these new nano-materials, a full structural characterisation using synchrotron radiation has been carried out. We present here the results of EXAFS, WAXS and High Energy X-ray Diffraction (HED) measurements. EXAFS gives the local coordination geometry of the metal atoms and the short-range metal-metal distances. WAXS gives the overall lattice structure and an independent measurement of metal-metal distances extending to longer range. HED focuses on structural anisotropy.

\section{Experimental}

\subsection{Preparation of nanoporous alumina membranes}

The nanoporous alumina membranes are fabricated by anodising high purity aluminium in polyprotic acids $[3,7]$. The thickness, of the order of several microns, can be controlled by of the length of the process. The pores are perpendicular to the membrane surface, packed in an ordered hexagonal array with wall thickness comparable to pore diameter. The pore diameters are monodisperse within the 5-200 nm range and controllable as a function of the constant anodising voltage applied. In our synthetic procedure, pore diameters above $12 \mathrm{~nm}$ are linearly related to anode voltage $(1.2 \mathrm{~nm} / \mathrm{V})$. Smaller pores, which would be most desirable as hosts for quantum wires, show a more complex relationship of size to growth voltage, and a less regular pore structure. 
Table 1. Preparation conditions of the metal nanowires.

\begin{tabular}{ccc}
\hline Metal & Electrolyte & Voltage \\
\hline $\mathrm{Au}$ & $1 \mathrm{~g} / \mathrm{l} \mathrm{HAuCl}_{4}+20 \mathrm{~g} / \mathrm{l} \mathrm{MgSO}_{4} \cdot 7 \mathrm{H}_{2} \mathrm{O}, \mathrm{pH} 1.7$ & 9 \\
$\mathrm{Ag}$ & $1 \mathrm{~g} / \mathrm{lgNO}_{3}+41 \mathrm{~g} / \mathrm{l} \mathrm{MgSO} 4 \cdot 7 \mathrm{H}_{2} \mathrm{O}, \mathrm{H}_{2} \mathrm{SO}_{4}, \mathrm{pH} 2$ & 9 \\
$\mathrm{Cu}$ & $35 \mathrm{~g} / \mathrm{l} \mathrm{CuSO}_{4}+20 \mathrm{~g} / \mathrm{lgSO}_{4} \cdot 7 \mathrm{H}_{2} \mathrm{O}, \mathrm{H}_{2} \mathrm{SO}_{4}, \mathrm{pH} 1.2$ & 9 \\
$\mathrm{Fe}$ & $120 \mathrm{~g} / \mathrm{l} \mathrm{FeSO}_{4} \cdot 7 \mathrm{H}_{2} \mathrm{O}+45 \mathrm{~g} / \mathrm{l} \mathrm{H}_{3} \mathrm{BO}_{3}, 1 \mathrm{~g} / 1$ ascorbic acid & 16 \\
$\mathrm{Co}$ & $50 \mathrm{~g} / \mathrm{CoSO}_{4} \cdot 7 \mathrm{H}_{2} \mathrm{O}+25 \mathrm{~g} / \mathrm{l} \mathrm{H}_{3} \mathrm{BO}_{3}, 20 \mathrm{~g} / 1$ glycerine & 16 \\
\hline
\end{tabular}

Pore diameters are measured by electron microscopy of ultramicrotomed films, but a more complete characterisation of pore diameter and geometry has been performed using SAXS. These data are currently under analysis [9].

\subsection{Preparation of metal nanowires}

Metal nanowires are produced within the nanoporous alumina membranes. The pores are filled with different metals $(\mathrm{Au}, \mathrm{Ag}, \mathrm{Cu}, \mathrm{Fe}, \mathrm{Co})$ using an electrochemical AC plating procedure with an appropriate electrolyte [7] (cf. Table 1). Because the membrane acts as a rectifier, the amount of metal plated during the cathodic half-cycle is larger than the amount of metal that is reoxidised during the anodic half-cycle. This results in net metal plating. AC is used rather than DC in order to prevent the system from being polarised.

Since the membranes provide up to $10^{11}$ pores per $\mathrm{cm}^{2}$ that are perpendicular to the surface and nearly parallel to each other, up to $10^{11}$ metallic nanowires per $\mathrm{cm}^{2}$ can be prepared using this method.

After plating the metal, the aluminium can be dissolved from the back of the alumina film by a $10 \%$ solution of $\mathrm{Br}_{2}$ in methanol. This is necessary for diffraction measurements, where the aluminium lattice would dominate diffraction from the metal nanowires; but not for EXAFS, which is an element-specific technique.

\subsection{Synchrotron measurements}

EXAFS data were collected at CLRC SRS (Daresbury, UK) on beamlines 7.1 and 9.2. Measurements were made in fluorescence mode using a 13-channel signal detector. Membranes were stacked in order to produce a suitable edge jump. Some brittle membranes (including those with the aluminium backing removed) were ground with boron nitride and pressed into pellets. Samples were cooled to $80 \mathrm{~K}$ with a cold finger liquid-nitrogen cryostat. EXAFS data were collected up to $k=20 \AA^{-1}$. Signal/noise ratios were improved by summing several datasets.

WAXS data were collected at ESRF (Grenoble, France) on beamline ID01 operating under beam conditions of $6 \mathrm{GeV}, 90 \mathrm{~mA}, 16$-bunch mode using an X-ray energy of $8.00 \mathrm{keV}$, corresponding to a wavelength of $1.54 \AA$. Data were collected at sample temperatures of $298 \mathrm{~K}$. The
Table 2. Representative EXAFS nearest-neighbour distances and Debye-Waller factors for metal nanowires in alumina pores of the stated diameters.

\begin{tabular}{llllll}
\hline Metal & pore & lattice & $R / \AA$ & $2 \sigma^{2} / \AA^{-2}$ & $R$-factor \\
\hline $\mathrm{Au}$ & $48 \mathrm{~nm}$ & fcc & $2.869(3)$ & $0.0073(1)$ & $21.0 \%$ \\
$\mathrm{Ag}$ & $48 \mathrm{~nm}$ & fcc & $2.878(2)$ & $0.0093(5)$ & $21.2 \%$ \\
$\mathrm{Cu}$ & $24 \mathrm{~nm}$ & fcc & $2.544(3)$ & $0.007(1)$ & $30.0 \%$ \\
$\mathrm{Fe}$ & $24 \mathrm{~nm}$ & bcc & $2.479(3)$ & $0.005(1)$ & $27.0 \%$ \\
$\mathrm{Co}$ & $12 \mathrm{~nm}$ & fcc & $2.497(3)$ & $0.012(1)$ & $24.0 \%$ \\
$\mathrm{Co}$ & $48 \mathrm{~nm}$ & hcp & $2.500(3)$ & $0.005(1)$ & $19.0 \%$ \\
\hline
\end{tabular}

crystallite diameters were calculated from the widths of the (111) peaks using the Scherrer formula.

High Energy X-ray Diffraction measurements were made at ESRF (Grenoble, France) on beamline ID15B operating under beam conditions of $6 \mathrm{GeV}, 80 \mathrm{~mA}, 16$-bunch mode using an X-ray energy of $90 \mathrm{keV}$, corresponding to a wavelength of $0.13689 \AA$. This station is fitted with a $2 \mathrm{D}$ image-plate detector giving the azimuthal variation of the diffraction pattern up to a k-value of above $25 \AA^{-1}$. The membranes were studied mounted on a special sample holder allowing a full rotation ( $\phi$-variation) of the samples by 360 degrees. They were rotated in the main beam to present a varying angle of the nanowire axis to the scattering vector, including membrane positions face-on and edge-on to the incident beam. Data were collected at sample temperatures of $298 \mathrm{~K}$.

\section{Results and discussion}

Nearest-neighbour metal-metal distances and lattice structures, obtained from analysis of the EXAFS and WAXS data, are summarised in Table 2.

Because ordinary diffraction measurements cannot see clearly the anisotropy of the structure, HED was capable of giving much more valuable information than WAXS on the preferred orientation. When the membrane was faceon to the beam (the nanowire axis parallel to the beam) no preferred orientation could be seen. The data presented in this paper were collected with the membrane edge-on to the incident beam (nanowire axis perpendicular to the beam). 


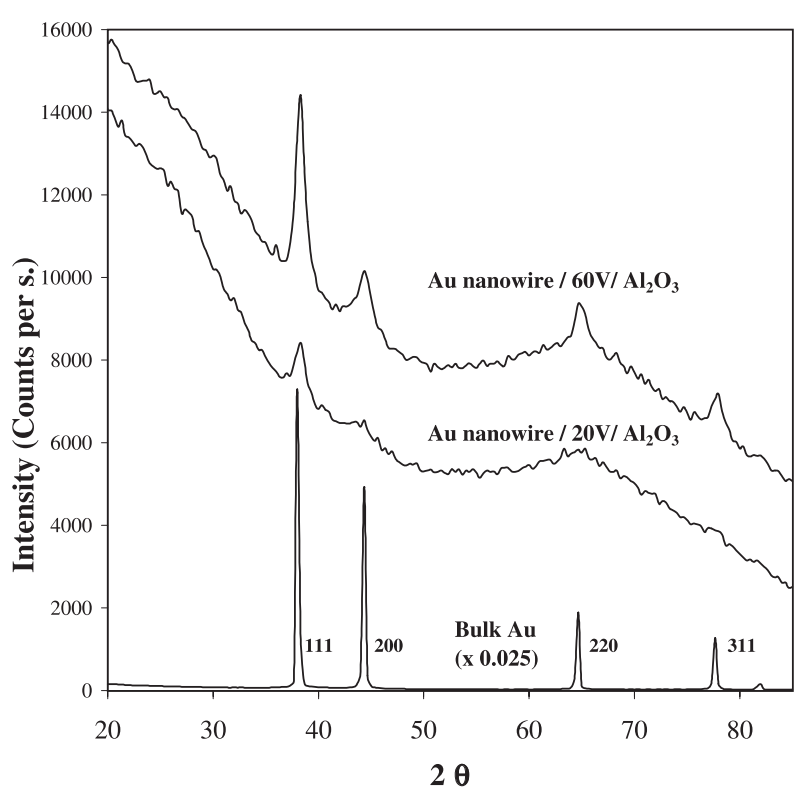

Fig. 1. WAXS patterns (ESRF beamline ID01) of gold nanowires in $20 \mathrm{~V}$ and $60 \mathrm{~V}$ alumina membranes, with the pattern from bulk gold for comparison.

The very high quality of these HED patterns taken with the image plate detector on ESRF beamline ID15B demonstrates the remarkable capability of this equipment in terms of the characterisation of preferred orientation. The results are exceptional considering the very low metal content of these samples (less than $1 \%$ by weight).

\subsection{Gold nanowires}

Nanowires grown in 20, 40 and 60V membranes were investigated. Gold nanowires were found to have the same fcc structure and $\mathrm{Au}-\mathrm{Au}$ distance as the bulk metal, but with smaller EXAFS Debye-Waller factors indicating a higher degree of crystallinity [10]. Figure 1 shows the WAXS patterns of nanowires in the $20 \mathrm{~V}$ and the $60 \mathrm{~V}$ membranes.

The study of the fwhm of the (111) reflection of the WAXS data showed that crystallites in the membranes with 24 and $72 \mathrm{~nm}$ pore diameter have an elongated shape of respectively 7 and $9 \mathrm{~nm}$ diameter. Several crystallites occupy the pore diameter. The high-energy diffraction data showed no sign of the presence of preferred orientation.

X-ray absorption near-edge spectroscopy (XANES) has shown the electronic structure of these nanowires to be intermediate between that of Au clusters and bulk Au [10].

\subsection{Silver nanowires}

Nanowires grown in 4, 8, 10, 40 and $60 \mathrm{~V}$ membranes were investigated. Silver nanowires adopt the same fcc structure as their bulk form but with higher crystallinity as shown by the EXAFS Debye-Waller factors. The crystallites in the small pores of diameter 9.6 and $12 \mathrm{~nm}$ have

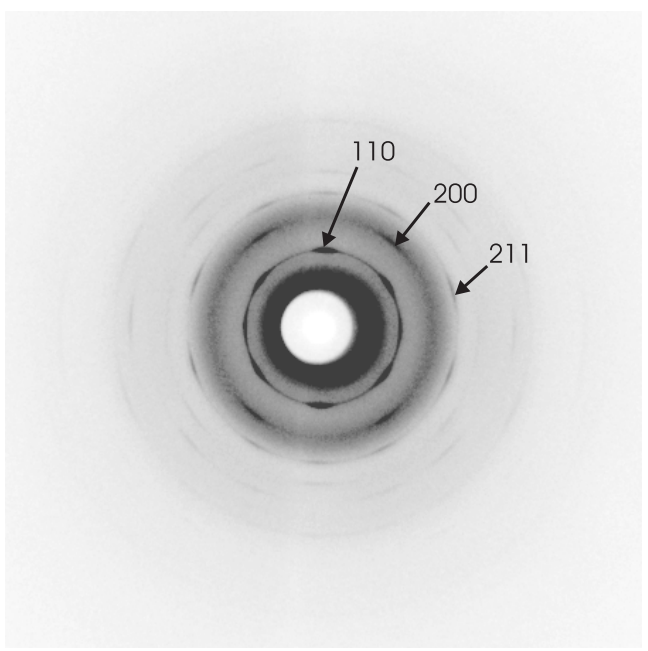

Fig. 2. High-energy diffraction pattern (ESRF beamline ID15B) of Fe nanowires in alumina with $24 \mathrm{~nm}$ pore diameter.

roughly the same diameter as the pore, respectively 8.6 and $12.6 \mathrm{~nm}$. In the bigger pores of 48 and $72 \mathrm{~nm}$ diameter, crystallites adopt an elongated shape of respectively 25 and $11 \mathrm{~nm}$ diameter with several crystallites occupying the pore diameter. A slight preferred orientation with the [110] direction perpendicular to the pore axis was found for the $72 \mathrm{~nm}$ pores. For the $4 \mathrm{~V}$ membrane, the Ag crystallite size was $12 \mathrm{~nm}$, showing clearly that for the smallest pore size the metal has not grown confined within the pores.

\subsection{Copper nanowires}

EXAFS and WAXS showed that $\mathrm{Cu}$ nanowires grown in $20 \mathrm{~V}$ membranes adopt the same fcc structure as bulk $\mathrm{Cu}$. Crystallites of $12 \mathrm{~nm}$ diameter occupy the $24 \mathrm{~nm}$ pore diameter. Only a very slight preferred orientation of the crystallites could be seen in the HED pattern, with the [110] direction perpendicular to the axis of the wire.

\subsection{Iron nanowires}

Fe nanowires grown in $10 \mathrm{~V}$ and $20 \mathrm{~V}$ membranes were investigated. EXAFS and HED both showed that iron nanowires in pores of diameter $12 \mathrm{~nm}$ and $24 \mathrm{~nm}$ are structurally similar to bcc bulk iron. However their XANES shows significant differences from the bulk. The EXAFS structural analysis and the XANES present no sign of oxidation, a result confirmed by Mössbauer spectroscopy [8].

Figure 2 shows the HED pattern of Fe nanowires in alumina with $24 \mathrm{~nm}$ pore diameter. The nanowires are made of crystallites of $7.8 \mathrm{~nm}$ diameter. A strong preferred orientation with the [110] or [111] directions perpendicular to the pore axis can clearly be seen on this pattern.

These Fe nanowires have been found from susceptibility measurements and Mössbauer spectroscopy to have unusual anisotropic magnetic properties [8]. Our results give a structural explanation for these properties. 


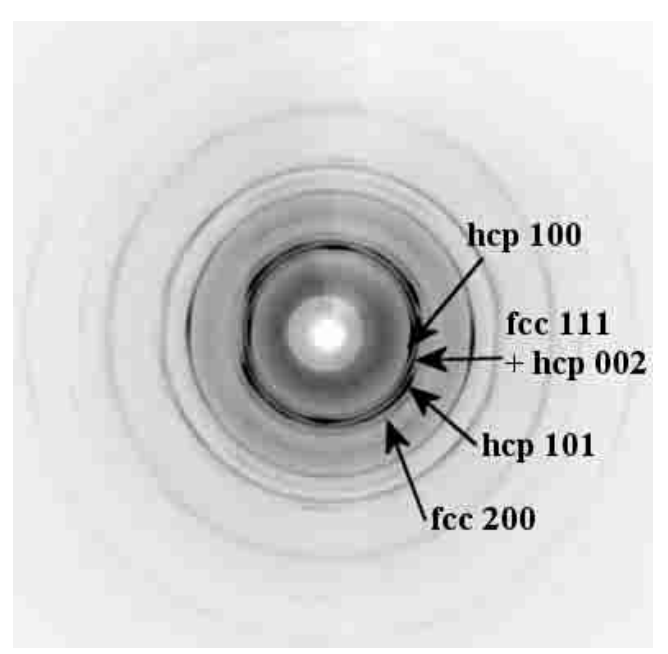

Fig. 3. HED pattern (ESRF beamline ID15B) of Co nanowires in alumina with $48 \mathrm{~nm}$ pore diameter.

\subsection{Cobalt nanowires}

Unusual anisotropic magnetic behaviour has been reported for cobalt nanowires [8]. As the magnetic measurements showed differences according to the pore size, two Co nano-wires grown in $10 \mathrm{~V}$ and $40 \mathrm{~V}$ membranes were investigated. Diffraction measurements showed that both cobalt nanowires were composed of a mixture of two different phases: the hcp phase, stable at room temperature, and the fcc high temperature $\beta$-form of the cobalt structure that is normally only stable above $417^{\circ} \mathrm{C}$ [11]. For the bigger pore of $48 \mathrm{~nm}$ diameter, the hcp phase formed the majority whilst for the smaller pore size $12 \mathrm{~nm}$ the main phase present seemed to be the fcc phase.

The high energy diffraction pattern of the $48 \mathrm{~nm}$ pore diameter is presented in Figure 3. Moreover, in the $48 \mathrm{~nm}$ pore we found a very strong preferred orientation with the [100] direction perpendicular to the pore axis for both fcc and hcp structures. The preferred phase and orientation both seem to be very sensitive to the pore diameter. The cobalt nanowires are composed of crystallites of spherical shape filling completely the diameter in the $12 \mathrm{~nm}$ pores and of $19 \mathrm{~nm}$ diameter in the $48 \mathrm{~nm}$ pores.

It was recently concluded [12] from ${ }^{59} \mathrm{Co}$ NMR spectroscopy that cobalt nanowires in commercially available porous alumina films of $20 \mathrm{~nm}$ and $100 \mathrm{~nm}$ pore diameter consist of a mixture of fcc and hcp phases with the unique axis of the hcp phase oriented preferentially perpendicular to the wires. Our diffraction results are a direct structural confirmation of the mixture of phases, but show a different preferred orientation, raising the possibility that this is sensitive to the pore diameter and growth conditions.

\section{Conclusions}

Using EXAFS, WAXS and High Energy X-ray Diffraction, we have structurally characterised nanowires of gold, silver, copper, iron and cobalt confined within nanoporous alumina membranes.
The metal nanowires are polycrystalline and composed of crystallites showing a great variation of size, shape and orientation according to the metal type and the pore size.

The gold, silver, copper and iron nanowires adopt the same structure as their bulk forms but present a different crystallinity. For the cobalt nanowires, a mixture of fcc and hcp phases is observed in a ratio depending on the alumina pore sizes, with fcc (normally stable only at high temperatures) preferred in small pore size.

The sensitivity to pore size of the phase type and/or preferred orientation of $\mathrm{Fe}$ and $\mathrm{Co}$ nanowires, which we have demonstrated by our structural studies, is the key to understanding their magnetic behaviour.

The confinement of these metal nanowires within the alumina membranes seems to confer on them very unusual properties and structure. The use of these alumina membranes as templates to prepare new types of nanomaterials looks very promising.

Our work is part of the EU TMR network CLUPOS which is studying the potential application of these materials in electronic devices [6]. For financial support we thank the EU TMR programme contract number FMRX-CT98-0177. We also wish to acknowledge the use of the EPSRC's Chemical Database Service at Daresbury [13].

\section{References}

1. R.L. Whetten, M.N. Shafigullin, J.T. Khoury, T.G. Schaaff, I. Vezmar, M.M. Alvarez, A. Wilkinson, Acc. Chem. Res. 32, 397 (1999).

2. J.S. Yin, Z.L. Wang, Nanostruct. Mat. 11, 845 (1999).

3. C.A. Foss, G.L. Hornyak, J.A. Stockert, C.R. Martin, J. Phys. Chem 98, 2963 (1994).

4. T. Kyotani, B.K. Pradhan, A. Tomita, Bull. Chem. Soc. Jap. 72, 1957 (1999).

5. G. Hornyak, M. Kröll, R. Pugin, T. Sawitowski, G. Schmid, J.-O. Bovin, G. Karsson, H. Hofmeister, S. Hopfe, Chem. Eur. J. 3, 1951 (1997).

6. Research Network homepage: www.clupos.1th.se

7. T.-A. Hanaoka, A. Heilmann, M. Kröll, H.-P. Kormann, T. Sawitowski, G. Schmid, P. Jutzi, A. Klipp, U. Kreibig, R. Neuendorf, Appl. Organometal. Chem. 12, 367 (1998).

8. P. Paulus, F. Luis, M. Kröll, G. Schmid, L.J. De Jongh, J. Magn. Magn. Mater. 224, 180 (2001).

9. D. Grandjean, J.C. Dore, R.E. Benfield, M. Kröll, G. Schmid, D. Le Bolloc'h, submitted for publication.

10. R.E. Benfield, D. Grandjean, M. Kröll, R. Pugin, T. Sawitowski, G. Schmid, J. Phys. Chem. B 105, 1961 (2001).

11. N.N. Greenwood, A. Earnshaw, Chemistry of the Elements (Pergamon, Oxford, 1984), p. 1292.

12. G.J. Strijkers, J.H.J. Dalderop, M.A.A. Broeksteeg, H.J.M. Swagten, W.J.M. de Jonge, J. Appl. Phys. 86, 5141 (1999).

13. The United Kingdom Chemical Database Service: D.A. Fletcher, R.F. McMeeking, D.J. Parkin, Chem. Inf. Comput. Sci. 36, 746 (1996). 